\title{
Symmetric hyperbolic system in the Ashtekar formulation
}

\author{
Gen Yonedal \\ Department of Mathematical Science, Waseda University, Okubo 3-4-1, Shinjuku, Tokyo 169-8790, Japan \\ Hisa-aki Shinkait \\ Department of Physics, Washington University, St. Louis, MO 63130-4899, USA
}

(submitted March 24, 1998; revised Sep. 23, 1998)

\begin{abstract}
We present a first-order symmetric hyperbolic system in the Ashtekar formulation of general relativity for vacuum spacetime. We add terms from the constraint equations to the evolution equations with appropriate combinations, which is the same technique used by Iriondo, Leguizamón and Reula ( Phys. Rev. Lett. 79, 4732 (1997) ). However our system is different from theirs in the points that we primarily use Hermiticity of a characteristic matrix of the system to characterize our system symmetric, discuss the consistency of this system with reality condition, and show the characteristic speeds of the system.
\end{abstract}

PACS numbers: 04.20.Cv, 04.20.Ex, 04.20.Fy

\section{INTRODUCTION}

Hyperbolic formulation of the Einstein equation is the one of the main research areas in general relativity [1]. This formulation is used in the proof of the existence, uniqueness and stability (well-posedness) of the solutions of the Einstein equation by analytical methods [2]. So far, several first order hyperbolic formulations are proposed; some of them are flux conservative [3], symmetrizable [4], or symmetric hyperbolic system [5]. The recent interest in hyperbolic formulation arises from its application to numerical relativity. One of the expected advantages is the existence of the characteristic speeds of the system, with which we may treat the numerical boundary with appropriate condition. Some numerical tests have been reported along this direction 810 ].

Recently, Iriondo, Leguizamón and Reula (ILR) 11 discuss a symmetric hyperbolic system in the Ashtekar formulation [12] of general relativity. Ashtekar's formulation has many advantages in the treatment of gravity. By using his special pair of variables, the constraint equations which appear in the theory become low-order polynomials, and the theory has the correct form for gauge theoretical features. These suggest possibilities for treating a quantum description of gravity nonperturbatively. The classical applications of the Ashtekar's formulation has also been discussed by several authors. For example, we [14] discussed the reality conditions for metric and triad and proposed new set of variables from the point of Lorentzian dynamics. We [15] also showed an example of passing degenerate point in 3-space by loosing reality condition locally.

In this Letter, we present a new symmetric hyperbolic system in the Ashtekar's formulation for Lorentzian vacuum spacetime. ILR 11 says that they construct a symmetric hyperbolic system. However, we think their discussion is not clear in the the following three points. First, they used anti-Hermiticity of the pricipal symbol for defining their system symmetric. We, however, think that this does not derive Hermiticity of the characteristic matrix [ $A$ below in eq. (3.1), $B$ in [11]], since they do not define their vector $k_{a}$ explicitly. We rather use Hermiticity of the characteristic matrix primarily to construct a symmetric hyperbolic system. Second, they did not mention the consistency of their formulation with the reality conditions which are crucial in the study of the Lorentzian dynamics in the Ashtekar variables [19]. Third, they did not discuss the characteristic structure of the system, which should be shown in the normal hyperbolic formulations. Our discussion covers these two matters.

The construction of this paper is as follows. After giving a brief review of Ashtekar's variables and reality conditions in $\S 2$, we present our formulation in $\S 3$. The discussion of characteristic speed and summary are in $\S 4$.

\section{ASHTEKAR'S FORMULATION}

The key feature of Ashtekar's formulation of general relativity 12 is the introduction of a self-dual connection as one of the basic dynamical variables. Let us write the metric $g_{\mu \nu}$ using the tetrad, $e_{\mu}^{I}$, and define its inverse, $E_{I}^{\mu}$, by $g_{\mu \nu}=e_{\mu}^{I} e_{\nu}^{J} \eta_{I J}$ and $E_{I}^{\mu}:=e_{\nu}^{J} g^{\mu \nu} \eta_{I J}$. We

\footnotetext{
* We use $\mu, \nu=0, \cdots, 3$ and $i, j=1, \cdots, 3$ as spacetime indices, while $I, J=(0), \cdots,(3)$ and $a, b=(1), \cdots,(3)$ are $S O(1,3), S O(3)$ indices respectively. We raise and lower $\mu, \nu, \cdots$ by $g^{\mu \nu}$ and $g_{\mu \nu}$ (Lorentzian metric); $I, J, \cdots$ by $\eta^{I J}=$ $\operatorname{diag}(-1,1,1,1)$ and $\eta_{I J} ; i, j, \cdots$ by $\gamma^{i j}$ and $\gamma_{i j}(3$-metric $)$. We use volume forms $\epsilon_{a b c} ; \epsilon_{a b c} \epsilon^{a b c}=3$ !.
} 
define $\mathrm{SO}(3, \mathrm{C})$ self-dual and anti self-dual connections ${ }^{ \pm} \mathcal{A}_{\mu}^{a}:=\omega_{\mu}^{0 a} \mp(i / 2) \epsilon_{b c}^{a} \omega_{\mu}^{b c}$, where $\omega_{\mu}^{I J}$ is a spin connection 1-form (Ricci connection), $\omega_{\mu}^{I J}:=E^{I \nu} \nabla_{\mu} e_{\nu}^{J}$. Ashtekar's plan is to use only a self-dual part of the connection ${ }^{+} \mathcal{A}_{\mu}^{a}$ and to use its spatial part ${ }^{+} \mathcal{A}_{i}^{a}$ as a dynamical variable. Hereafter, we simply denote ${ }^{+} \mathcal{A}_{\mu}^{a}$ as $\mathcal{A}_{\mu}^{a}$.

The lapse function, $N$, and shift vector, $N^{i}$, are expressed as $E_{0}^{\mu}=\left(1 / N,-N^{i} / N\right)$. This allows us to think of $E_{0}^{\mu}$ as a normal vector field to $\Sigma$ spanned by the condition $t=x^{0}=$ const., which plays the same role as that of ADM. Ashtekar treated the set $\left(\mathcal{A}_{i}^{a}, \tilde{E}_{a}^{i}\right)$ as basic dynamical variables, where $\tilde{E}_{a}^{i}$ is an inverse of the densitized triad defined by $\tilde{E}_{a}^{i}:=e E_{a}^{i}$ where $e:=\operatorname{det} e_{i}^{a}$ is a density. This pair forms the canonical set.

In the case of pure gravitational spacetime, the Hilbert action takes the form

$$
\begin{aligned}
S= & \int \mathrm{d}^{4} x\left[\left(\partial_{t} \mathcal{A}_{i}^{a}\right) \tilde{E}_{a}^{i}+(i / 2) \underset{\sim}{N} \tilde{E}_{a}^{i} \tilde{E}_{b}^{j} F_{i j}^{c} \epsilon_{c}^{a b}-\Lambda \underset{\sim}{N} \operatorname{det} \tilde{E}\right. \\
& \left.-N^{i} F_{i j}^{a} \tilde{E}_{a}^{j}+\mathcal{A}_{0}^{a} \mathcal{D}_{i} \tilde{E}_{a}^{i}\right],
\end{aligned}
$$

where $N:=e^{-1} N, \Lambda$ is the cosmological constant, $\mathcal{D}_{i} \tilde{E}_{a}^{i}:=\partial_{i} \tilde{E}_{a}^{i}-i \epsilon_{a b}{ }^{c} \mathcal{A}_{i}^{b} \tilde{E}_{c}^{i}$, and $\operatorname{det} \tilde{E}$ is defined to be $\operatorname{det} \tilde{E}=(1 / 6) \epsilon^{a b c} \epsilon_{\sim} i j k \tilde{E}_{a}^{i} \tilde{E}_{b}^{j} \tilde{E}_{c}^{k}$, where $\epsilon_{i j k}:=\epsilon_{a b c} e_{i}^{a} e_{j}^{b} e_{k}^{c}$ and $\underset{\sim}{\epsilon} i_{k}:=e^{-1} \epsilon_{i j k}$ 门。.

Varying the action with respect to the non-dynamical variables $\underset{\sim}{N}, N^{i}$ and $\mathcal{A}_{0}^{a}$ yields the constraint equations,

$$
\begin{aligned}
\mathcal{C}_{H} & :=(i / 2) \epsilon_{c}^{a b} \tilde{E}_{a}^{i} \tilde{E}_{b}^{j} F_{i j}^{c}-\Lambda \operatorname{det} \tilde{E} \approx 0, \\
\mathcal{C}_{M i} & :=-F_{i j}^{a} \tilde{E}_{a}^{j} \approx 0, \\
\mathcal{C}_{G a} & :=\mathcal{D}_{i} \tilde{E}_{a}^{i} \approx 0,
\end{aligned}
$$

where $F_{\mu \nu}^{a}:=\left(d \mathcal{A}^{a}\right)_{\mu \nu}-(i / 2) \epsilon^{a}{ }_{b c}\left(\mathcal{A}^{b} \wedge \mathcal{A}^{c}\right)_{\mu \nu}$ is the curvature 2 -form.

The equations of motion for the dynamical variables $\left(\mathcal{A}_{i}^{a}\right.$ and $\left.\tilde{E}_{a}^{i}\right)$ are

$$
\begin{aligned}
& \partial_{t} \mathcal{A}_{i}^{a}=-i \epsilon^{a b}{ }_{c}{ }_{\sim} \tilde{E}_{b}^{j} F_{i j}^{c}+N^{j} F_{j i}^{a}+\mathcal{D}_{i} \mathcal{A}_{0}^{a}+e \Lambda \underset{\sim}{N} e_{i}^{a}, \\
& \partial_{t} \tilde{E}_{a}^{i}=-i \mathcal{D}_{j}\left(\epsilon^{c b}{ }_{a}{ }_{\sim} \tilde{E}_{c}^{j} \tilde{E}_{b}^{i}\right)+2 \mathcal{D}_{j}\left(N^{[j} \tilde{E}_{a}^{i]}\right)+i \mathcal{A}_{0}^{b} \epsilon_{a b}{ }^{c} \tilde{E}_{c}^{i}
\end{aligned}
$$

where $\mathcal{D}_{j} X_{a}^{j i}:=\partial_{j} X_{a}^{j i}-i \epsilon_{a b}{ }^{c} \mathcal{A}_{j}^{b} X_{c}^{j i}$, for $X_{a}^{i j}+X_{a}^{j i}=0$.

In order to construct metric variables from the variables $\left(\mathcal{A}_{i}^{a}, \tilde{E}_{a}^{i}, \underset{\sim}{N}, N^{i}\right)$, we first prepare tetrad $E_{I}^{\mu}$ as $E_{0}^{\mu}=\left(1 / \underset{\sim}{N},-N^{i} / e \underset{\sim}{N}\right)$ and $E_{a}^{\mu}=\left(0, \tilde{E}_{a}^{i} / e\right)$. Using them, we obtain metric $g^{\mu \nu}$ such that

$$
g^{\mu \nu}:=E_{I}^{\mu} E_{J}^{\nu} \eta^{I J}
$$

$$
{ }^{\dagger} \epsilon_{x y z}=e, \underset{\sim}{\epsilon_{x y z}}=1, \epsilon^{x y z}=e^{-1}, \tilde{\epsilon}^{x y z}=1 .
$$

Notice that in general the metric (2.7) is not real. To ensure the metric is real-valued, we need to impose real lapse and shift vectors together with two conditions (metric reality condition);

$$
\begin{aligned}
\operatorname{Im}\left(\tilde{E}_{a}^{i} \tilde{E}^{j a}\right) & =0, \\
\operatorname{Re}\left(\epsilon^{a b c} \tilde{E}_{a}^{k} \tilde{E}_{b}^{(i} \mathcal{D}_{k} \tilde{E}_{c}^{j)}\right) & =0,
\end{aligned}
$$

where the latter comes from the secondary condition of reality of the metric $\operatorname{Im}\left\{\partial_{t}\left(\tilde{E}_{a}^{i} \tilde{E}^{j a}\right)\right\}=0$ [13], and we assume $\operatorname{det} \tilde{E}>0$ (see 14).

For later convenience, we also prepare stronger reality conditions. These conditions are

$$
\begin{aligned}
\operatorname{Im}\left(\tilde{E}_{a}^{i}\right) & =0 \\
\text { and } \operatorname{Im}\left(\partial_{t} \tilde{E}_{a}^{i}\right) & =0,
\end{aligned}
$$

and we call them the "primary triad reality condition" and the "secondary triad reality condition", respectively. Using the equations of motion of $\tilde{E}_{a}^{i}$, the gauge constraint (2.4), the metric reality conditions (2.8), (2.9) and the primary condition (2.10), we see that (2.11) is equivalent to [14]

$$
\operatorname{Re}\left(\mathcal{A}_{0}^{a}\right)=\partial_{i}(\underset{\sim}{N}) \tilde{E}^{i a}+(1 / 2 e) e_{i}^{b} \underset{\sim}{N} \tilde{E}^{j a} \partial_{j} \tilde{E}_{b}^{i}+N^{i} \operatorname{Re}\left(\mathcal{A}_{i}^{a}\right),
$$

or with un-densitized variables,

$$
\operatorname{Re}\left(\mathcal{A}_{0}^{a}\right)=\partial_{i}(N) E^{i a}+N^{i} \operatorname{Re}\left(\mathcal{A}_{i}^{a}\right) .
$$

From this expression we see that the second triad reality condition restricts the three components of "triad lapse" vector $\mathcal{A}_{0}^{a}$. Therefore $(2.12)$ is not a restriction on the dynamical variables $\left(\mathcal{A}_{i}^{a}\right.$ and $\left.\tilde{E}_{a}^{i}\right)$ but on the slicing, which we should impose on each hypersurface. Thus the second triad reality condition does not restrict the dynamical variables any further than the second metric condition does.

\section{HYPERBOLIC FORMULATION}

We start from defining hyperbolic system following Friedrichs [16], which is first applied in general relativity by Fischer and Marsden [5]. That is, we say that the system is first-order (quasi-linear) hyperbolic if a certain pair of variables $u_{i}$ form a linear system as

$$
\partial_{t} u_{i}=A^{l j}{ }_{i}(u) \partial_{l} u_{j}+B_{i}(u),
$$

where $A$ is a characteristic matrix-valued function, of which eigenvalues are all real, and $B$ is a function. We further define that the system is symmetric when $A$ is a Hermitian matrix [6]17].

The symmetric system gives us the energy integral inequalities, which are the primary tools for analizing wellposedness of the system. As was discussed by Geroch 
[18], most physical systems are expressed as symmetric hyperbolic systems.

Ashtekar's formulation itself is in the first-order form in the sence of (3.1), but not a symmetric hyperbolic form.

We start from writing the principal part of the Ashtekar's evolution equations as

$$
\partial_{t}\left[\begin{array}{c}
\tilde{E}_{a}^{i} \\
\mathcal{A}_{i}^{a}
\end{array}\right] \cong\left[\begin{array}{cc}
A^{l}{ }_{a}^{b i}{ }_{j} & B_{a b}^{l i j} \\
C^{l a b}{ }_{i j} & D^{l a}{ }_{b i}{ }^{i j}
\end{array}\right] \partial_{l}\left[\begin{array}{c}
\tilde{E}_{b}^{j} \\
\mathcal{A}_{j}^{b}
\end{array}\right]
$$

where $\cong$ means that we extracted only the terms which appear in the principal part of the system. The system is symmetric hyperbolic if

$$
\begin{aligned}
& 0=A^{l a b i j}-\bar{A}^{l b a j i}, \\
& 0=D^{l a b i j}-\bar{D}^{l b a i j}, \\
& 0=B^{l a b i j}-\bar{C}^{l b a j i} .
\end{aligned}
$$

where bar denotes complex conjugate. ${ }^{\ddagger}$

We first prepare the constraints (2.2)-(2.4) as

$$
\begin{aligned}
\mathcal{C}_{H} & \cong i \epsilon^{a b}{ }_{c} \tilde{E}_{a}^{i} \tilde{E}_{b}^{j} \partial_{i} \mathcal{A}_{j}^{c}=i \epsilon_{b}^{d c} \tilde{E}_{d}^{l} \tilde{E}_{c}^{j}\left(\partial_{l} \mathcal{A}_{j}^{b}\right) \\
& =-i \epsilon_{b}{ }^{c d} \tilde{E}_{c}^{j} \tilde{E}_{d}^{l}\left(\partial_{l} \mathcal{A}_{j}^{b}\right), \\
\mathcal{C}_{M k} & =-F_{k j}^{a} \tilde{E}_{a}^{j} \cong-\left(\partial_{k} \mathcal{A}_{j}^{a}-\partial_{j} \mathcal{A}_{k}^{a}\right) \tilde{E}_{a}^{j} \\
& =\left[-\delta_{k}^{l} \tilde{E}_{b}^{j}+\delta_{k}^{j} \tilde{E}_{b}^{l}\right]\left(\partial_{l} \mathcal{A}_{j}^{b}\right), \\
\mathcal{C}_{G a} & =\mathcal{D}_{i} \tilde{E}_{a}^{i} \cong \partial_{l} \tilde{E}_{a}^{l} .
\end{aligned}
$$

We apply the same technique with ILR to modify the equation of motion of $\tilde{E}_{a}^{i}$ and $\mathcal{A}_{i}^{a}$ by adding the constraints which weakly produce $\mathcal{C}_{H}=0, \mathcal{C}_{M k}=0$, and $\mathcal{C}_{G a}=0$. With a parametrization for triad lapse $\mathcal{A}_{0}^{a}$ with $T$ and $S$ as

$$
\partial_{i} \mathcal{A}_{0}^{a} \cong T^{l}{ }_{i j}^{a b} \partial_{l} \tilde{E}_{b}^{j}+S_{i{ }_{b}{ }^{a j}} \partial_{l} \mathcal{A}_{j}^{b},
$$

we write the principal parts of (2.5) and (2.6) as

$$
\begin{aligned}
\partial_{t} \tilde{E}_{a}^{i}= & -i \mathcal{D}_{j}\left(\epsilon^{c b}{ }_{a} N \tilde{E}_{c}^{j} \tilde{E}_{b}^{i}\right)+2 \mathcal{D}_{j}\left(N^{[j} \tilde{E}_{a}^{i]}\right) \\
& +i \mathcal{A}_{0}^{b} \epsilon_{a b}{ }^{c} \tilde{E}_{c}^{i}+P^{i}{ }_{a b} \mathcal{C}_{G}^{b} \\
\cong & -i \epsilon^{c b}{ }_{a}{ }_{\sim}\left(\partial_{j} \tilde{E}_{c}^{j}\right) \tilde{E}_{b}^{i}-i \epsilon^{c b}{ }_{a} N \tilde{E}_{c}^{j}\left(\partial_{j} \tilde{E}_{b}^{i}\right) \\
& +\mathcal{D}_{j}\left(N^{j} \tilde{E}_{a}^{i}\right)-\mathcal{D}_{j}\left(N^{i} \tilde{E}_{a}^{j}\right)+P^{i}{ }_{a}{ }^{b} \partial_{j} \tilde{E}_{b}^{j} \\
\cong & {\left[-i \epsilon^{b c}{ }_{a} N \delta_{j}^{l} \tilde{E}_{c}^{i}-i \epsilon^{c b}{ }_{a} N \tilde{E}_{c}^{l} \delta_{j}^{i}\right.} \\
& \left.+N^{l} \delta_{j}^{i} \delta_{a}^{b}-N^{i} \delta_{j}^{l} \delta_{a}^{b}+P_{a}^{i b}{ }_{j} \delta_{j}^{l}\right]\left(\partial_{l} \tilde{E}_{b}^{j}\right),
\end{aligned}
$$

$\partial_{t} \mathcal{A}_{i}^{a}=-i \epsilon^{a b}{ }_{c}{ }_{\sim} \tilde{E}_{b}^{j} F_{i j}^{c}+N^{j} F_{j i}^{a}$

\footnotetext{
${ }^{\ddagger}$ We think that the reader will not confuse $A^{l a b i j}$ and $B^{l a b i j}$ with matrix $A$ and $B$ in (3.1).
}

$$
\begin{aligned}
& +\mathcal{D}_{i} \mathcal{A}_{0}^{a}+e \Lambda \underset{\sim}{N} e_{i}^{a}+Q_{i}^{a} \mathcal{C}_{H}+R_{i}^{j a} \mathcal{C}_{M j} \\
& \cong-i \epsilon^{a b}{ }_{c}{ }_{\sim} \tilde{E}_{b}^{j}\left(\partial_{i} \mathcal{A}_{j}^{c}-\partial_{j} \mathcal{A}_{i}^{c}\right)+N^{j}\left(\partial_{j} \mathcal{A}_{i}^{a}-\partial_{i} \mathcal{A}_{j}^{a}\right)
\end{aligned}
$$

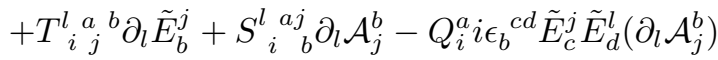

$$
\begin{aligned}
& +R_{i}^{k a}\left[-\delta_{k}^{l} \tilde{E}_{b}^{j}+\delta_{k}^{j} \tilde{E}_{b}^{l}\right] \partial_{l} \mathcal{A}_{j}^{b} \\
& \cong\left[+i \epsilon_{b}^{a}{ }^{c}{ }_{\sim} \tilde{E}_{c}^{j} \delta_{i}^{l}-i \epsilon_{b}^{a}{ }^{c}{ }_{\sim}^{N} \tilde{E}_{c}^{l} \delta_{i}^{j}+N^{l} \delta_{b}^{a} \delta_{i}^{j}-N^{j} \delta_{b}^{a} \delta_{i}^{l}\right. \\
& \left.+S^{l}{ }_{i}^{a j}{ }_{b}-i Q_{i}^{a} \epsilon_{b}{ }^{c d} \tilde{E}_{c}^{j} \tilde{E}_{d}^{l}-R_{i}{ }^{l a} \tilde{E}_{b}^{j}+R_{i}{ }^{j a} \tilde{E}_{b}^{l}\right]\left(\partial_{l} \mathcal{A}_{j}^{b}\right) \\
& +T_{i j}^{l a b} \partial_{l} \tilde{E}_{b}^{j},
\end{aligned}
$$

where $P, Q$ and $R$ are parameters and will be fixed later. Note that we truncated $\mathcal{A}_{0}^{a}$ in $(3.10)$, while it remains in (3.11), since only the derivative of $\mathcal{A}_{0}^{a}$ effects the principal part of the system. From these two equations, we get

$$
\begin{aligned}
A^{l a b i j}= & -i \epsilon^{b c a} \underset{\sim}{N} \gamma^{l j} \tilde{E}_{c}^{i}-i \epsilon^{c b a} \underset{\sim}{N} \tilde{E}_{c}^{l} \gamma^{i j} \\
& +N^{l} \gamma^{i j} \delta^{a b}-N^{i} \gamma^{l j} \delta^{a b}+P^{i a b} \gamma^{l j}, \\
B^{l a b i j}= & 0, \\
C^{l a b i j}= & T^{l i a j b}, \\
D^{l a b i j}= & +i \epsilon^{a b c} \underset{\sim}{N} \tilde{E}_{c}^{j} \gamma^{l i}-i \epsilon^{a b c}{\underset{\sim}{N}}_{E_{c}^{l}} \gamma^{j i} \\
& +N^{l} \delta^{a b} \gamma^{j i}-N^{j} \delta^{a b} \gamma^{l i}+S^{l i a j b} \\
& -i Q^{a i} \epsilon^{b c d} \tilde{E}_{c}^{j} \tilde{E}_{d}^{l}-R^{i l a} \tilde{E}^{j b}+R^{i j a} \tilde{E}^{l b} .
\end{aligned}
$$

The condition (3.3) is written as

$$
\begin{aligned}
0= & -i \epsilon^{b c a} \underset{\sim}{N} \gamma^{l j} \tilde{E}_{c}^{i}-i \epsilon^{a c b} \underset{\sim}{N} \gamma^{l i} \overline{\tilde{E}}_{c}^{j} \\
& -2 i \epsilon^{c b a} \underset{\sim}{\operatorname{Im}}\left(\tilde{E}_{c}^{l}\right) \gamma^{i j}-N^{i} \gamma^{l j} \delta^{a b} \\
& +N^{j} \gamma^{l i} \delta^{b a}+P^{i a b} \gamma^{l j}-\bar{P}^{j b a} \gamma^{l i} .
\end{aligned}
$$

Because the third term in the right-hand-side cannot be elliminated using $P$, we assume the triad reality condition $\operatorname{Im}\left(\tilde{E}_{c}^{l}\right)=0$ hereafter. Then (3.4) and (3.5) become

$$
\begin{aligned}
0= & \bar{T}^{l j b i a} \\
0= & i \epsilon^{a b c} \underset{\sim}{N} \tilde{E}_{c}^{j} \gamma^{l i}+i \epsilon^{b a c} \underset{\sim}{N} \tilde{E}_{c}^{i} \gamma^{l j}-N^{j} \delta^{a b} \gamma^{l i}+N^{i} \delta^{b a} \gamma^{l j} \\
& +S^{l i a j b}-\bar{S}^{l j b i a}-i Q^{a i} \epsilon^{b c d} \tilde{E}_{c}^{j} \tilde{E}_{d}^{l}-i \bar{Q}^{b j} \epsilon^{a c d} \tilde{E}_{c}^{i} \tilde{E}_{d}^{l} \\
& -R^{i l a} \tilde{E}^{j b}+R^{i j a} \tilde{E}^{l b}+\bar{R}^{j l b} \tilde{E}^{i a}-\bar{R}^{j i b} \tilde{E}^{l a} .
\end{aligned}
$$

The third and forth term in (3.18) cannot be elliminated using $Q$ or $R$, so $S^{l i a j b}=\gamma^{l i} \delta^{a b} N^{j}$ is determined. Thus $S$ and $T^{l j b i a}=0$ [eq. (3.17)] decides the form of the triad lapse as

$$
\mathcal{A}_{0}^{a}=\mathcal{A}_{j}^{a} N^{j}+\text { non-dynamical terms }
$$

in result. In order to be consistent with the triad lapse condition (2.13), we need to specify the lapse as $\partial_{i} N=$ 0 . This lapse condition is also supported by the fact that if we do not assume $\partial_{i} N=0$, then the secondary triad reality condition (2.12) makes the system second order. ILR does not discuss consistency of the system 
with reality condition (especially with secondary reality condition). However, since ILR assume $\mathcal{A}_{0}^{a}=\mathcal{A}_{j}^{a} N^{j}$, we think that ILR also needs to impose similar restricted lapse condition in order to preserve reality of the system.

The rest of our effort is finished when we specify parameters $P, Q$ and $R . P$ is given by decomposing (3.16) into real/complex parts;

$$
\begin{aligned}
0= & -N^{i} \gamma^{l j} \delta^{a b}+N^{j} \gamma^{l i} \delta^{b a} \\
& +\operatorname{Re}(P)^{i a b} \gamma^{l j}-\operatorname{Re}(P)^{j b a} \gamma^{l i} \\
0= & -\epsilon^{b c a} \underset{\sim}{N} \gamma^{l j} \tilde{E}_{c}^{i}-\epsilon^{a c b} \underset{\sim}{N} \gamma^{l i} \tilde{E}_{c}^{j} \\
& +\operatorname{Im}(P)^{i a b} \gamma^{l j}+\operatorname{Im}(P)^{j b a} \gamma^{l i}
\end{aligned}
$$

By multiplying $\gamma_{l i}$ in these two and taking symmetric and anti-symmetric operation to the index $a b$, we obtain

$$
P^{i a b}=N^{i} \delta^{a b}+i \underset{\sim}{N} \epsilon^{a b c} \tilde{E}_{c}^{i} .
$$

For $Q$ and $R$, we found that a combination of the choice

$$
\begin{aligned}
Q^{a i} & =e^{-2} \underset{\sim}{N} \tilde{E}^{i a} \\
R^{i l a} & =i e^{-2} \underset{\sim}{N} \epsilon^{a c d} \tilde{E}_{d}^{i} \tilde{E}_{c}^{l}
\end{aligned}
$$

satisfies the condition (3.18).

\section{DISCUSSION}

In summary, by adding constraint terms with appropriate coefficients, we succeed to construct a symmetric hyperbolic formulation for the Ashtekar's system. This formulation is consistent with secondary triad reality condition, which requires to impose a constant lapse function for the evolving system.

The characteristic speeds of this system are given by finding eigenvalues of the characteristic matrix $A$ of (3.1). Since $A$ is a Hermitian, eigenvalues of $A$ are all real. Then it is again clear that this system is symmetric hyperbolic. Actually the eigenvalues of the $18 \times 18$ matrix $A^{l}$ for $x^{l}$-direction are: $N^{l}$ (multiplicity $\left.=6\right), N^{l} \pm \sqrt{\gamma^{l l}} N(5$ each), and $N^{l} \pm 3 \sqrt{\gamma^{l l}} N$ (1 each), where we do not take the sum in $\gamma^{l l}$ here. These speeds are independent from the way of taking a triad. We omit to show the related eigen-vectors because of saving space.

As we denoted in $\S 3$, our formulation requires triad reality condition. In order to make the system first order, the lapse function is assumed to be constant. Shift vectors and triad lapse $\mathcal{A}_{0}^{a}$ should have a relation (3.19). This can be interpreted that shift is free and triad lapse is determined. This gauge restriction sounds tight, but this arises from our general assumption of (3.9). There might be a possibility to improve the situation by renormalizing shift and triad lapse terms into left-hand-side of equations of motion like the case of GR [4]. Or this might be because our system is constituted by Ashtekar's original variables. We are now trying to release this gauge restriction and/or to simplify the characteristic speeds by other gauge possiblities and also by introducing new dynamical variables. This effort will be reported elsewhere.

This work (HS) was partially supported by NSF PHYS 96-00049, 96-00507, and NASA NCCS 5-153.

ฯ To appear in Phys. Rev. Lett. (gr-qc/9803077)

† Electronic address: yoneda@mn.waseda.ac.jp

¥ Electronic address: shinkai@null.wustl.edu

[1] Recent review is given by O. A. Reula, Living Rev. Relativity, 98-3, (1998).

[2] Y. Choquet-Bruhat and J.W. York. Jr., in General Relativity and Gravitation, vol. 1, ed. by Held., (Plenum, New York, 1980).

[3] C. Bona, J. Massó, E. Seidel, J. Stela, Phys. Rev. Lett. 75, 600 (1995); Phys. Rev. D56, 3405 (1997).

[4] Y. Choquet-Bruhat and J.W. York, C. R. Acad. Sci. Paris, t. 321, Série I, 1089 (1995); A. Abrahams, A. Anderson, Y. Choquet-Bruhat and J.W. York, Phys. Rev. Lett. 75, 3377 (1995).

[5] A. E. Fischer and J. E. Marsden, Commun. Math. Phys. 28, p1-38 (1972)

[6] H. Friedrich, Class. Quantum Grav. 13, 1451 (1996).

[7] S. Frittelli, O.A. Reula, Phys. Rev. Lett. 76, 4667 (1996).

[8] M. Alcubierre, Phys. Rev. D55, 5981 (1997).

[9] M.A. Scheel, T.W. Baumbarte, G.B. Cook, S.L. Shapiro, and S.A. Teukolsky, Phys. Rev. D56 6320 (1997); ibid. D58, 044020 (1998).

[10] C. Bona, J. Massó, E. Seidel and P. Walker, grqc/9804052.

[11] M.S. Iriondo, E.O. Leguizamón, O.A. Reula, Phys. Rev. Lett. 79, 4732 (1997).

[12] A. Ashtekar, Phys. Rev. Lett. 57, 2244 (1986); Phys. Rev. D36, 1587 (1987); Lectures on Non-Perturbative Canonical Gravity (Singapore, World Scientific, 1991).

[13] A. Ashtekar, J. D. Romano and R. S. Tate, Phys. Rev. D40, 2572 (1989).

[14] G. Yoneda and H. Shinkai, Class. Quantum Grav. 13, 783 (1996).

[15] G. Yoneda, H. Shinkai and A. Nakamichi, Phys. Rev. D56, 2086 (1997).

[16] K. O. Friedrichs, Comm. Pure and Appl. Math., 7, 345 (1954).

[17] R. Courant and D. Hilbert, Methods of Mathematical Physics, Volume II, (John Willey \& Sons, 1962)

[18] R. Geroch, Partial Differential Equations in Physics, grqc/9602055

[19] After we submitted this paper and to gr-qc/9803077, we found that ILR put their second paper at gr-qc/9804019. In the second paper, they discuss how to treat reality condition in the causal region in different way than we do. 